\title{
Evaluation of patients' adherence to chemotherapy for breast cancer
}

\author{
Adewale O Adisa, Olukayode O Lawal, Abdul RK Adesunkanmi \\ Department of Surgery, Obafemi Awolowo University Teaching Hospitals Complex, Ile-Ife,
} Nigeria.

Correspondence to: Dr. Adewale O. Adisa; Department of Surgery;Obafemi Awolowo University Teaching Hospitals Complex, Ile-Ife, Nigeria. E-mail: wadisc@yahoo.com. Tel: +2348033889425

\section{SUMMARY}

The study aimed to establish the common reasons for non-adherence to drug treatment among breast cancer patients at the Obafemi Awolowo University Teaching Hospitals Complex, Ile-Ife, Nigeria over a ten-year period (Jan 1993 Dec 2002). Patients' adherence and reasons for non-adherence to chemotherapy were evaluated using descriptive statistics. Two hundred and twenty-five breast cancer patients including 199 females and 6 males with breast cancer were recruited. $12(5.3 \%)$ patients had Stage I disease while $126(56 \%)$ were in stage IV. The non-adherence rate was $80.9 \%$ and $111(73 \%)$ of the non-adherent patients were eventually not seen again. Of the one hundred and one patients who gave reasons for non-adherence, $45 \%$ complained of financial difficulties; $18 \%$ thought they were well enough; $15 \%$ were fearful of subsequent operation and $11 \%$ were unable to further bear the drug side effects. In conclusion, the study demonstrated poor economic status of the patients as one of the major reasons for non-adherence to cancer chemotherapy in Nigeria. Government subsidy of breast cancer treatment, improved health education and advocacy complemented by home visiting to encourage hospital attendance would be required for better adherence to chemotherapy.

[Afr J Health Sci. 2008; 15:22-27]

\section{Introduction}

The burden of breast cancer in developing countries is enormous [1]. A twofold increase has been reported in the incidence in Ibadan, Nigeria and in Kampala, Uganda in the recent past $[2,3]$. A more aggressive disease course has also been noted as reported from Jos in northern Nigeria where most patients presented with high grade invasive ductal carcinoma which were oestrogen receptor (ER) and progesterone receptor (PR) negative but p53 positive [4]. The patients also commonly present late, with advanced disease and are frequently lost to follow up early in management with poor overall survival rate in different centres across Africa $[1,5,9]$.

The major role of chemotherapy in the management of breast cancer and its efficacy in prolonging survival has been well documented [10-13]. The beneficial effects of chemotherapeutic agents can only be realised if patients comply with their use. Compared with the developed countries, patients' compliance with chemotherapy in developing countries has been shown to be poorer [14-17].

More recently, the more authorityladen term "compliance" is largely being replaced with the term "adherence" in the literature due to increase in patient autonomy. Many health care providers prefer the word "adherence", because "compliance" suggests that the patient is passively following the doctor's orders and that the treatment plan is not based on an established therapeutic agreement between the patient and the physician The most commonly cited definition of adherence is 'the extent to which a person's behaviour coincides with medical or health advice'. [18,19] The aim of this study was to determine the level of adherence to chemotherapy as well as factors that may 
influence this among breast cancer patients in our environment.

\section{Materials and Methods}

A review of the clinical records of histologically proven cases of breast cancer seen over a ten year period of January 1993 through December 2002 at the Ife Hospital Unit of the Obafemi Awolowo University Teaching Hospitals Complex (OAUTHC), IleIfe, South-West Nigeria was carried out. Those whose hospital records were incomplete were excluded. Demographic and clinical information including treatment records were studied. Chemotherapeutic agents received, the numbers of courses, patient's adherence as well as details of non-adherence were further studied.

A patient was considered to be nonadherent if they did not present for two consecutive doses of chemotherapy. Patients who had dose reduction and/ or omission due to inadequate haematological profile or poor clinical condition were not included as defaulters. The patients who missed their medications due to no fault of theirs either because of unplanned public holidays or other reasons beyond their control were excluded from the study. The data were subjected to descriptive analyses.

\section{Results}

There were 225 cases of breast cancer seen during the study period. Majority (97.3\%) were females and 6 were males. The ages ranged from 26 to 86 years with a mean of 48 (SD: 12.3) years. More than one quarter of the patients were under 40years of age, with $69(30.6 \%)$ being 40-49years old, and $13(5.8 \%) 70 y e a r s$ and above. There were more lesions on the right $(47.1 \%)$ than the left (44.1\%). Twenty patients $(8.9 \%)$ had bilateral breast involvement at presentation. Using the American Joint Committee on Cancer (AJCC) staging system, $12(5.3 \%)$ patients presented in Stage I, 28 (12.4\%) in stage II, $59(26.2 \%)$ in stage III and $126(56 \%)$ in stage IV (Table I). Presentation at advanced stage was recorded for all age groups.

Twenty-six patients who presented in very poor clinical condition died before chemotherapy could be instituted while another eleven refused chemotherapy even after adequate counselling. On the whole, 188 of the 225 patients had chemotherapy. Of these, $59 \quad(31.4 \%)$ had neo-adjuvant chemotherapy alone, $81(43.1 \%)$ had adjuvant chemotherapy while 48 (25.5\%) had both neoadjuvant and adjuvant chemotherapy. CMF combination chemotherapy (Cyclophosphamide, Methotrexate and 5Fluorouracil) was used in 149 patients, while CAF (Cyclophosphamide, Adriamycin and 5Fluorouracil) was used in combination for 21 patients. CMF with Vincristine (CMFV) was used in 12 patients. 92 (48.9\%) patients had Tamoxifen prescribed along with their chemotherapy. (Table II)

The non-adherence rate was $80.9 \%$ (152 of 188) and a pattern of non-adherence was seen across all age groups, chemotherapy combinations and disease stage. Forty $(21.3 \%)$ patients defaulted before the end of the first course while $95(50.5 \%)$ patients defaulted within the first three courses of chemotherapy. Also, we found that at one year after defaulting, 111(73\%) of these patients had not returned for further treatment and many were subsequently lost to follow up. Only 7 patients came back to continue treatment within a month of defaulting.

The reasons for non-adherence were available in only 101 cases. Of these, 45 $(45 \%)$ were noted to have defaulted due to financial constraints, $18(18 \%)$ patients because they felt well; 15 (15\%) because of fear of subsequent operation; 11 (11\%) did so because of unbearable drug side effects, while $19(19 \%)$ patients defaulted for various other reasons.

The group of patients $(\mathrm{N}=18)$ who were non-adherent because they felt well were aged between 30-45years and 16 of them had stage I and II diseases. Nine had neo-adjuvant chemotherapy with very good response and indeed 5 out of them had a dramatic response with non-palpable tumours after the first course of chemotherapy. The remaining 9 had mastectomy but defaulted before, during or after the first course of adjuvant chemotherapy. Twelve patients of this group were lost to follow up while 6 re-presented again with stage IV disease.

In the final analysis, 51patients $(22.6 \%)$ died during the course of treatment, $22(9.8 \%)$ patients were referred for radiotherapy, $15(6.7 \%)$ re-presented with 
recurrence after initial treatment, 5 (2.3\%) were adjudged to be cured of the disease (having attended follow-up clinic for over 5years without any recurrence), while 132 $(58.7 \%)$ patients were lost to follow-up.

Table 1: Age and Stage at Presentation of Women with Breast Cancer in Ile-Ife, Nigeria.

Age

(Yr)

$<30$

30-39

40-49

$50-59$

60-69

70-79

$\geq 80$
Stage at Presentation

$\begin{array}{cccc}\text { StageI } & \text { StageII } & \text { StageIII } & \text { StageIV } \\ 2 & - & 1 & 3 \\ 1 & 6 & 14 & 29 \\ 4 & 11 & 14 & 40 \\ 4 & 7 & 15 & 23 \\ 1 & 3 & 8 & 26 \\ - & 1 & 6 & 4 \\ - & - & 1 & 1\end{array}$

Table 2: Use of Chemotherapy in Women with Breast Cancer in Ile-Ife, Nigeria.

$\begin{array}{llcccr}\text { Drugs Used } & \text { No }(\%) & \text { Neo-Adjuvant } & \text { Adjuvant } & \\ \text { CMF } & 149(79.3 \%) & 112 & 52 & \\ \text { CAF } & 21(11.2 \%) & 9 & & 12 & \\ \text { CAMF } & 12(6.4 \%) & 2 & 1 & & 5 \\ \text { CMFV } & 6(3.2 \%) & & 1 & 42 & \end{array}$

Table 3: Reasons for Non-Adherence with Chemotherapy Schedule

$\begin{array}{lcccc}\text { Reason } & \text { No } & & \text { Percentage } \\ \text { Financial Difficulty } & 45 & & 45 \% & \\ \text { Feels Well } & 18 & & 18 \% & \\ \text { Fear of Operation } & 15 & & 15 \% & \\ \text { Drug Side Effects } & 11 & & 11 \% & \\ \text { Others } & & 19 & & 19 \%\end{array}$

(No reasons were given for non-adherence in 51 patients)

\section{Discussion}

The findings in this study suggesting a high incidence of breast cancer in young women with more than one quarter of the patients being below 40years of age has been the experience of others in previous studies across Nigeria $[7,8,20]$. The reason why breast cancer tends to occur in younger age groups in Africans is not yet known. It may however be related to the young population of most of the African countries just like other developing countries.

The problem of late presentation in the hospital, a common phenomenon among patients in developing countries, was also observed in this study. One hundred and twenty-six patients $(56 \%)$ presented with stage IV disease. Similar findings have also been reported in many studies on breast cancer across Nigeria and in other developing countries $[7-9,21,22]$. This may be due to poor awareness of the disease among the population as well as low level of organised public enlightenment and screening programmes in most developing countries. Okobia et al reported a poor knowledge of breast cancer among rural dwelling women in Nigeria with only a minority practising breast self examination and clinical breast examination [20]. A study in South Africa found that $77 \%$ of black women presented with Stages III and IV disease while only a few who reportedly practised self breast examination presented with stage I disease [23].

In view of the late stages of presentation, chemotherapy played a major role in the management of the patients. In this study more than $83 \%$ of the patients had chemotherapy as neo-adjuvant or adjuvant treatment or a combination of both. 81 patients had neo-adjuvant chemotherapy to downstage 
the tumour preparatory for surgery while 130 patients had adjuvant chemotherapy. This is largely due to the fact that a large number of the patients presented late thus precluding initial surgery. Combination chemotherapy was used in all the patients. The combination of Cyclophosphamide, Methotrexate and 5Fluorouracil (CMF) was used in most of the patients in this study. Anthracycline-based combinations, which are common in developed countries, were used in only $11 \%$ of the patients in this study largely due to its high cost. Similarly patients were not placed on newer chemotherapeutic agents such as the Taxanes as they were largely unavailable and unaffordable to most of our patients.

Since there is no health welfare scheme in place in Nigeria during the period of the study, the burden of procuring expensive chemotherapeutic agents was borne by the patients and their relations. Significant proportion of the patients being agrarian and generally poor, could not afford the estimated average cost of US $\$ 100$ for procuring a course of CMF chemotherapy. This probably affected patients' adherence to the chemotherapy schedule in most instances. Indeed $45 \%$ of patients who gave reasons for non-adherence to chemotherapy cited financial constraint as the problem. In the experience of the authors, many of the patients in the environment are unable to procure more than the first course of chemotherapy and are usually not able to continue with the schedule. Perhaps if cancer treatment were to be subsidized again by the government (as was the case in the early 1980s in our part of the country) perhaps there would be significant improvement in the rate of nonadherence.

In a study of patients adherence to chemotherapy for breast cancer carried out at Sussex, United Kingdom, 55\% of patients receiving chemotherapy were noted not to have adhered to medications either unintentionally (due largely to forgetfulness) or intentionally (due to drug side effects and difficulty swallowing tablets) [24]. Drug side effects have also been given as reason for nonadherence among women taking Tamoxifen $[25,26]$. In the current series, 11 patients $(5.8 \%)$ reported intentional non-adherence because of drug side effects. Some of these were young women who had severe alopecia and skin hyperpigmentation which were cosmetically unacceptable. Two of them had recurrent nausea and vomiting that necessitated admission for rehydration and they subsequently defaulted.

It is of interest that 15 patients defaulted after counselling for mastectomy. In Africa, many women find it difficult to accept the option of mastectomy [27]. A study in Egypt observed that most of the women were primarily concerned with keeping their breasts regardless of the disease stage [28]. It is possible that the relatively young age of many of the women in this study, lack of understanding by their spouses, the general fear of surgery and anaesthesia as well as the relatively low rate of breast reconstruction surgeries were responsible for not readily consenting to mastectomy. Furthermore because most of the patients present with advanced disease, the option of breast conserving surgeries is not usually available for them.

It is also worthy of note that the group of 18 patients that defaulted for reason of being better were young adults with operable disease stages. One half of them were on neoadjuvant chemotherapy with significant effects including complete regression in five patients. The paradox of wellness and non-adherence is a difficult problem, as these patients would probably have benefited more from chemotherapy use. It is possible that religious belief in faith healing that is common in the environment and the initial tumour disappearance led to default based on bad advice.

The clinic attendance by the patients was also a problem. More than one-half (132, representing $58.7 \%$ ) of the patients were lost to follow-up at different points in the course of their treatment. This observation is similar to findings in many studies from developing countries [29-30]. Problems confronting the patients ranging from financial difficulties to the prolonged denial of the illness as well as the strong superstitious belief in the use of traditional and or spiritual healing approaches may contribute to this. The poor follow-up made it difficult to assess the efficacy of chemotherapy as well as the final outcome in many of the patients. Health education and advocacy as well as home visiting and tracing of breast cancer patients may reduce this problem. 
Conclusion

This study has, in highlighting the severe burden of breast cancer in Nigeria demonstrated poor economic status of the patients as one of the major reasons for nonadherence to cancer chemotherapy. One way perhaps of encouraging patients to present and adhere to chemotherapy treatment is to introduce highly subsidized breast cancer treatment in government hospitals. Health education and advocacy complemented by home visiting to encourage hospital attendance may be required to improve the rate of adherence to chemotherapy.

\section{References}

1. Anim JT. Breast cancer in subSaharan African women. African Journal of Medicine and Medical Sciences. 1993; 22:5-10.

2. Wabinga HR, Parkin DM, WabwireMangen F, Nambooze S: Trends in cancer incidence in Kyadondo County, Uganda, 1960-1997.British Journal of Cancer. 2000; 82:1585-1592.

3. Adebamowo $\mathrm{CA}$ and Ajayi OO. Breast Cancer in Nigeria. West African Journal of Medicine. 2000; 19:179-191.

4. Gukas ID, Jennings BA, Mandong BM, Igun GO, Girling AC, Manasseh AN, Ugwu BT and Leinster SJ. Clinicopathological features and molecular markers of breast cancer in Jos, Nigeria. West African Journal of Medicine. 2005; 24:209-213.

5. Ikpatt OF, Ndoma-Egba R and Collan Y. Influence of age and prognosis of breast cancer in Nigeria. East African Medical Journal. 2002; 79:651-657

6. Adesunkanmi AR, Lawal OO, Adelusola KA and Durosimi MA. The severity, outcome and challenges of breast cancer in Nigeria. Breast. 2006; 15:399-409.

7. Anyanwu SN. Survival following treatment of primary breast cancer in eastern Nigeria. East African Medical Journal. 2000; 77:539-543.

8. Chiedozi LC. Breast carcinoma in young Nigerian women. Tropical
Geographical Medicine. 1984; 36:249-253.

9. Ihekwaba FN. The management of male breast cancer in Nigerians. Postgraduate Medical Journal. 1993; 69:562-565.

10. Eifel P, Axelson JA, Costa J, Crowley J, Curran WJ Jr., Deshler A, Fulton S, Hendricks CB, Kemeny M, Komblith AB, Louis TA, Markman M, Mayer R and Roter D. National institute of Health Consensus Development Conference Statement: adjuvant therapy for breast cancer, Nov 1-3, 2000. Journal of the National Cancer Institute. 2001.93:979-989

11. Adjuvant systemic therapy for women with node-negative breast cancer. The Steering Committee on Clinical Practice Guidelines for the Care and Treatment of Breast Cancer. Canadian Medical Association Journal. 1998; 158: S43-S51

12. Polychemotherapy for early breast cancer: an overview of the randomised trials. Early Breast Cancer Trialists' Collaborative Group. Lancet. 1998; 352:930-942.

13. Adjuvant systemic therapy for women with node-positive breast cancer. The Steering Committee on Clinical Practice Guidelines for the Care and Treatment of Breast Cancer. Canadian Medical Association Journal.1998; 158: S52-S64

14. Othieno-Abinya NA, Nyabola LO, Abwao HO and Ndege P. Postsurgical management of patients with breast cancer at Kenyatta National Hospital. East African Medical Journal. 2002; 79:156-162.

15. Taylor SE, Lichtman RR and Wood JV. Compliance with chemotherapy among breast cancer patients. Health Psychology. 1984; 3:553-562.

16. De Maio E, Gravina A, Pacilio C, Amabile G, Labonia V, Landi G, nuzzo F, Rossi E, D’Aluto G, Capasso I, Rinaldo M, Morrica B, Elmo M, Di Maio M, Perrone F and de Matteis A. Compliance and toxicity of adjuvant CMF in elderly breast cancer patients: a single-center experience. $B M C$ Cancer. 2005; 5:30 
17. Olivotto A, Coldman AJ, Hislop TG, Trevisan CH, Kula J, Goel V and Sawka C. Compliance with practice guidelines for node-negative breast cancer. Journal of Clinical Oncology. 1997; 15:216-222.

18. Steiner JF and Earnest MA. The language of medication-taking. Annals of Internal Medicine. 2000; 132:926930.

19. Osterberg $\mathrm{L}$ and Blaschke $\mathrm{T}$. Adherence to Medication. New England Journal of Medicine. 2005; 353:487-497

20. Okobia $\mathrm{MN}$, Bunker $\mathrm{CH}$, Okonofua FE and Osime U. Knowledge, attitude and practice of Nigerian women towards breast cancer: A crosssectional study. World Journal of Surgical Oncology. 2006; 4: 11.

21. Kidmas AT, Ugwu BT, Manasseh AN, Iya D and Opaluwa AS. Male breast malignancy in Jos University Teaching Hospital. West African Journal of Medicine. 2005; 24:36-40.

22. Ikpatt OF, Kuopio T, Ndoma-Egba R and Collan Y. Breast cancer in Nigeria and Finland: epidemiological, clinical and histological comparison. Anticancer Research. 2002; 22:30053012.

23. Vorobiof DA, Sitas F and Vorobiof G. Breast Cancer Incidence in South Africa. Journal of Clinical Oncology. 2001; 19:125S-127S

24. Atkins $\mathrm{L}$ and Fallowfield L. Intentional and non-intentional nonadherence to medication amongst breast cancer patients. European Journal of Cancer. 2006; 42:22712276

25. Grunfeld EA, Hunter MS, Sikka P and Mittal S. Adherence beliefs among breast cancer patients taking tamoxifen. Patient Educational and Counselling. 2005; 59:97-102.

26. Lash TL, Fox MP, Westrup JL, Fink AK and Silliman RA. Adherence to tamoxifen over the five-year course. Breast Cancer Research and Treatment. 2006; 99:215-220.

27. Ajekigbe AT. Fear of Mastectomy: the most common factor responsible for late presentation of carcinoma of the breast in Nigeria. Clinical Oncology
(Royal College of Radiologists). 1991; 3:78-80.

28. Nour A. Breast-conserving therapy in low-literacy patients in a developing country. Breast Journal. 2003; 9:7173

29. Nyirenda M, Sinfield R, Haves S, Molyneux EM and Graham SM. Poor attendance at a child TB contact clinic in Malawi. The International Journal of Tuberculosis and Lung Disease. 2006; 10:585-587

30. Yeats JR. Attendance compliance for short-course tuberculosis chemotherapy at clinics in Estcourt and surroundings. South African Medical Journal. 1986; 70:265-266. 